\title{
GDPs' confidence in treating emergency dental trauma
}

Management of dental trauma in primary care: a postal survey of general dental practitioners

\section{N. G. Jackson, P. J. Waterhouse and A. Maguire Br Dent J 2005; 198: 293-297}

Objective

To determine the self-perceived knowledge and attitudes of GDPs concerning management of dental trauma in primary care. To identify potential barriers to the management of dental trauma in primary care.

Design, setting and main outcome measures

A postal questionnaire survey of 417 GDPs in six local health authority districts in northeast England. Likert scale responses to 20 statements designed to test self-perceived knowledge and attitudes. Factor analysis with principle components analysis was undertaken to identify areas of correlation in questionnaire responses, followed by Chi squared test, Spearman's Rank Correlation and analysis of variance to measure association between variables.

\section{Results}

The response rate was 74\%. Enamel and dentine fractures were the most common injury, with 45\% of GDPs responding seeing more than 10 cases of dental trauma in the preceding year and 53\% of respondents seeing 1-3 cases of complicated crown fracture. Seventy-eight per cent believed that NHS remuneration was inadequate, but only $8 \%$ would refer dental trauma patients to secondary care for this reason. Half of the GDPs believed that trauma could be treated more effectively in practice if NHS payments were greater, and were more likely to agree with this statement if they had attended a course in treating dental trauma $(p=0.002)$. Single-handed GDPs were more likely to agree with the statements 'I would not treat dental trauma cases at my practice because the NHS payment is inadequate' $(p=0.008)$ and 'Treating dental trauma at my practice requires too much of my clinical time to be worthwhile' $(\mathrm{p}=0.002)$. The majority (96\%) of GDPs disagreed that treatment of dental trauma rested solely within secondary care and 96\% of GDPs agreed that they had a responsibility to provide initial emergency treatment for trauma patients prior to referral.

\section{Conclusions}

Although GDPs believed that financial remuneration was inadequate, it did not prevent them treating trauma cases. They agreed that they had responsibility for management of dental trauma in primary care and that it could be treated more effectively in practice if payment was greater. Time constraints were seen as a barrier to long-term management of complex trauma cases in primary care.

\section{IN BRIEF}

- An overview of the attitudes and beliefs of general dental practitioners (GDPs) with regard to the provision of treatment for traumatised teeth.

- Insight into the perceived level of confidence of GDPs in their ability to treat trauma cases.

- The barriers to care of trauma cases within the General Dental Service, as highlighted by GDPs themselves.

- Suggestion that communication between primary and secondary care providers could be improved, offering benefits to appropriate care provision.

\section{COMMENT}

By the age of 12 years, $12-33 \%$ of boys and $4-19 \%$ of girls will have suffered some dental trauma. The majority of these present at general practice where appropriate emergency care can make the difference between survival and loss of the pulp or even the tooth.

The General Dental Council ${ }^{1}$ requires all recent graduates to have knowledge of the management of trauma in both dentitions, but does not expect all to be experienced and competent in its clinical management. Recent advances in our understanding of pulp wound healing and techniques for vital pulp therapy are particularly relevant in this context, and it is possible that many caring general practitioners provide emergency trauma care without confidence that their interventions represent current best practice.

This carefully conducted questionnaire-based survey of general dentists in the North East of England enjoyed a 74\% response and provides robust observations of relevance to service development, care pathways, and continuing professional education.

General practitioners can lose out financially from the management of dental trauma, which must be accommodated at unpredictable times and can disrupt busy operating lists. Particular constraints are recognised when cases require long-term followup, but despite such tensions, the professional commitment to patient care over-rules for the vast majority of dentists and in most circumstances. Over $94 \%$ of respondents believed that general practitioners, not hospitals or other secondary care providers had responsibility to treat dental trauma, and $84 \%$ considered that effective care could be delivered in this setting.

While shouldering this responsibility, confidence in the care provided ranged from $99.7 \%$ for uncomplicated coronal fractures to $46 \%$ for avulsion injuries. Significant differences in outlook and confidence levels were noted between dentists who had recently attended a 'trauma' course and those who had not.

This manuscript highlights the need for proper financial recompense, improved access to continuing education and the development of decision-tree care pathways to inform correct decision making in trauma management. At a time of change in the delivery of primary dental services, ${ }^{2}$ it is clear that general practitioners should be supported in their efforts to provide prompt, high quality emergency care and long-term management for their trauma patients.

J. Whitworth,

School of Dental Sciences, Newcastle University doi: $10.1038 /$ sj.bdj.4812125

1. GDC. The first five years. A framework for undergraduate dental education. London, 2002.

2. Department of Health. Framework proposals for primary dental care services in England from 2005. London, 2004 